\title{
Doxorubicin-incorporated polymeric micelles composed of dextran-b-poly(DL-lactide-co-glycolide) copolymer
}

\author{
This article was published in the following Dove Press journal: \\ International Journal of Nanomedicine \\ 5 July 2011 \\ Number of times this article has been viewed
}

\author{
Young-II Jeongl,* \\ Do Hyung Kim ${ }^{1,2, *}$ \\ Chung-Wook Chung' \\ Jin-Ju Yoo' \\ Kyung $\mathrm{Ha} \mathrm{Choi}^{\prime}$ \\ Cy Hyun Kim ${ }^{1,2}$ \\ Seung Hee $\mathrm{Ha}^{\prime}$ \\ Dae Hwan Kang ${ }^{1,2}$
}

'National Research and Development Center for Hepatobiliary Cancer, Pusan National University Yangsan Hospital, Yangsan, Republic of Korea, Research Institute for Convergence of Biomedical Science and Technology, ${ }^{2}$ School of Medicine, Pusan National University, Yangsan, Republic of Korea

*These authors contributed equally to this work.
Correspondence: Dae Hwan Kang National Research and Development Center for Hepatobiliary Cancer, Pusan National University Yangsan Hospital, Beomeo-ri, Mulgeum-eup, Yangsan, Gyeongnam 626-770, Republic of Korea Tel +82 553603870

Fax +82 553603879

Emailsulsulpul@yahoo.co.kr
Background: Polymeric micelles using amphiphilic macromolecules are promising vehicles for antitumor targeting. In this study, we prepared anticancer agent-incorporated polymeric micelles using novel block copolymer.

Methods: We synthesized a block copolymer composed of dextran and poly (DL-lactide-co-glycolide) (DexbLG) for antitumor drug delivery. Doxorubicin was selected as the anticancer drug, and was incorporated into polymeric micelles by dialysis. Polymeric micelles were observed by transmission electron microscopy to be spherical and smaller than $100 \mathrm{~nm}$, with a narrow size distribution. The particle size of doxorubicin-incorporated polymeric micelles increased with increasing drug content. Higher initial drug feeding also increased the drug content.

Results: During the drug-release study, an initial burst release of doxorubicin was observed for 10 hours, and doxorubicin was continuously released over 4 days. To investigate the in vitro anticancer effects of the polymeric micelles, doxorubicin-resistant HuCC-T1 cells were treated with a very high concentration of doxorubicin. In an antiproliferation study, the polymeric micelles showed higher cytotoxicity to doxorubicin-resistant HuCC-T1 cells than free doxorubicin, indicating that the polymeric micelles were effectively engulfed by tumor cells, while free doxorubicin hardly penetrated the tumor cell membrane. On confocal laser scanning microscopy, free doxorubicin expressed very weak fluorescence intensity, while the polymeric micelles expressed strong red fluorescence. Furthermore, in flow cytometric analysis, fluorescence intensity of polymeric micelles was almost twice as high than with free doxorubicin.

Conclusion: DexbLG polymeric micelles incorporating doxorubicin are promising vehicles for antitumor drug targeting.

Keywords: dextran, polymeric micelle, block copolymer, poly(DL-lactide-co-glycolide)

\section{Introduction}

Dextran, a polysaccharide consisting of 1,6-, and 1,3-glucosidic linkages, is characterized as a colloidal and hydrophilic substance. Due to its biocompatibility and immunoneutrality, dextran is extensively employed as a bioinert material in biomedical science, tissue engineering, and drug delivery systems. ${ }^{1-5}$ In addition, dextran has confluent moieties for chemical conjugation, ie, a hydroxyl group exists in each glucose unit. Therefore, dextran is frequently investigated for use as a drug carrier system for anticancer drugs, antidiabetic agents, antibiotics, peptides, and enzymes. ${ }^{1-8}$

Block or graft copolymers consisting of hydrophilic and lipophilic domains are able to form via self-assembly in an aqueous environment into polymeric micelles, self-assembled nanoparticles, and core-shell-type nanoparticles. ${ }^{9-14}$ Among these 
configurations, polymeric micelles of block copolymers have unique properties, ie, the hydrophobic domain of the copolymer forms an inner core, and the hydrophilic domain forms a hydrated outer shell. ${ }^{12}$ The hydrophobic core is responsible for drug incorporation, and the hydrated outer shell guards the micelle from attack by the reticuloendothelial system. ${ }^{14}$ Polymeric micelles or core-shell-type block copolymer nanoparticles are attractive vehicles for solubilizing hydrophobic drugs, site-specific drug delivery via active or passive targeting mechanisms, reducing the amount of drug administered, and avoiding unwanted side effects. ${ }^{9,14}$ Previously, Jeong et al reported that poly(DL-lactide-co-glycolide) (PLGA)-grafted dextran copolymer is able to form self-assembling nanoparticles and can be used as a vehicle to carry antitumor agents. ${ }^{5}$ The dextran component of the copolymer forms the hydrophilic outer shell, due to its aqueous solubility, while PLGA forms the inner core of the nanoparticle.

In this study, we synthesized block copolymers composed of dextran and PLGA, and prepared polymeric micelles incorporating an anticancer agent for delivery. Because dextran has a hydrophilic domain and PLGA has a hydrophobic domain, dextran-PLGA block copolymer (DexbLG) may have amphiphilic properties in an aqueous environment. We expected that DexbLG block copolymer would be able to self-assemble as a core-shell-type polymeric micelle. Furthermore, we prepared polymeric micelles using a DexbLG block copolymer incorporating doxorubicin and evaluated their antitumor effects in vitro in cholangiocarcinoma cells.

\section{Methods and materials Materials}

Dextran from Leuconostoc spp (average molecular weight approximately 6000), triethylamine, doxorubicin $\mathrm{HCl}$, sodium cyanoborohydride, hexamethylene diamine, and thiazolyl blue tetrazolium bromide (MTT) were purchased from Sigma Chemical Company (St Louis, MO). N,N'dicyclohexyl carbodiimide and $\mathrm{N}$-hydroxysuccinimide were purchased from Aldrich Chemical Company (St Louis, MO). The dialysis membranes with molecular weight cutoffs of $2000 \mathrm{~g} / \mathrm{mol}$ and $8000 \mathrm{~g} / \mathrm{mol}$ were purchased from Spectra/PorTM dialysis membrane (Spectrum Laboratories Inc, Rancho Dominguez, CA). Dichloromethane and dimethyl sulfoxide were of high-performance liquid chromatography grade or extra pure grade. PLGA-500 (molecular weight $5000 \mathrm{~g} / \mathrm{mol}$ ) was purchased from Wako Pure Chemicals Company (Osaka, Japan).

\section{Synthesis of DexbLG block copolymer}

Aminated dextran was prepared as described by Maruyama et al ${ }^{15}$ with minor modifications. Dextran $180 \mathrm{mg}$ was dissolved in dimethyl sulfoxide, and sodium cyanoborohydride (189 mg, $3 \mathrm{mM}$ ) was added. This mixture was stirred for 24 hours at room temperature. Next, 10 equivalents of hexamethylene diamine were added, and the mixture was stirred for a further 24 hours at room temperature. The reactants were then introduced into the dialysis membrane (molecular weight cutoff, $2000 \mathrm{~g} / \mathrm{mol}$ ) and dialyzed against deionized water for 2 days. The dialyzed solution was lyophilized for 3 days. The conjugation yield of amine was determined by ${ }^{1} \mathrm{H}$ nuclear magnetic resonance (NMR) spectroscopy. ${ }^{16}$

N-hydroxysuccinimide PLGA (PLGA-NHS) was prepared as follows. PLGA $500 \mathrm{mg}$ was dissolved in dichloromethane, and 1.5 equivalents of $\mathrm{N}, \mathrm{N}^{\prime}$-dicyclohexyl carbodiimide and N-hydroxysuccinimide were added. This solution was stirred for 6 hours, and the reactants were filtered to remove byproducts. The solvent was removed under reduced pressure, and the solid was washed with methanol three times and dried under vacuum for 1 day.

For the DexLG block copolymer, $120 \mathrm{mg}$ of aminated dextran and $100 \mathrm{mg}$ of PLGA were dissolved in dry dimethyl sulfoxide. This solution was stirred in a nitrogen atmosphere for 2 days at room temperature. The reactants were then introduced into a dialysis membrane (molecular weight cutoff, $8000 \mathrm{~g} / \mathrm{mol}$ ) and dialyzed for 2 days against deionized water to remove the solvent and unreacted dextran. The dialyzed solution was lyophilized for 3 days. After that, the white solid harvested was added to dichloromethane to remove the unreacted PLGA, and the precipitants were harvested by filtration. The resulting white solid was dried under vacuum for 3 days. The mass of final product was determined, and the yield of product was determined to be $89 \%(\mathrm{w} / \mathrm{w})$ using the following equation:

Yield of product $(\%, \mathrm{w} / \mathrm{w})=[($ weight of final product $) /$

(weight of aminated dextran + PLGA-NHS) $] \times 100$.

\section{Fluorescence study}

To determine the critical micelle concentration of the DexbLG block copolymer, fluorescence spectroscopy (Shimadzu RF-5301 PC, Shimadzu Company Ltd, Japan) was performed, using pyrene as a hydrophobic probe as described previously. ${ }^{5}$ A polymeric micelle of DexbLG was prepared without incorporation of drug as follows. DexbLG $40 \mathrm{mg}$ was dissolved in $5 \mathrm{~mL}$ dimethyl sulfoxide and dialyzed against deionized water for 2 days. 
After that, the dialyzed solution was adjusted to various concentrations of polymeric micelles. To prepare sample solutions, a known amount of pyrene in acetone was added to each of a series of $20 \mathrm{~mL}$ vials, and the acetone was evaporated. The final concentration of pyrene was $6.0 \times 10^{-7} \mathrm{M}$, and $10 \mathrm{~mL}$ each of various concentrations of the polymeric micelle preparation were added and then heated for 3 hours at $65^{\circ} \mathrm{C}$. Equilibration of the pyrene and polymeric micelles was achieved by allowing the solutions to cool overnight at room temperature. Fluorescence excitation spectra were measured at an emission wavelength of $390 \mathrm{~nm}$. Excitation and emission bandwidths were both $1.5 \mathrm{~nm}$.

\section{Preparation of doxorubicin-incorporated polymeric micelles}

Polymeric micelles of the DexbLG block copolymer incorporating doxorubicin were prepared using the precipitationdialysis method. ${ }^{5}$ The DexbLG block copolymer was dissolved in $3 \mathrm{~mL}$ of dimethyl sulfoxide. Doxorubicin 5-10 mg was separately dissolved in $2 \mathrm{~mL}$ of dimethyl sulfoxide and then 20 microliters of triethylamine was added. This solution was added to the DexbLG/dimethyl sulfoxide solution and stirred magnetically for 1 hour. This solution was dropped into $15 \mathrm{~mL}$ of deionized water over 10 minutes to form the nanoparticles. The solvent was removed by dialysis, ie, the nanoparticle solution was introduced into the dialysis membrane (molecular weight cutoff $8000 \mathrm{~g} / \mathrm{mol}$ ) and dialyzed against distilled water for at least 1 day. During dialysis, the deionized water was exchanged at 1-2-hour intervals. The resulting solution was then used for analysis or lyophilized. Empty polymeric micelles of DexbLG block copolymer were prepared using the same procedure described above but without adding doxorubicin.

For evaluation of drug content and loading efficiency, $5 \mathrm{mg}$ of polymeric micelles incorporating doxorubicin were dissolved and diluted 100 -fold in $10 \mathrm{~mL}$ of dimethyl sulfoxide. The doxorubicin concentration was measured using an ultraviolet spectrophotometer (UV-1201, Shimadzu) at $479 \mathrm{~nm}$. Empty polymeric micelles of DexbLG block copolymer were used as a blank test. The following equations was used to determine drug content and loading efficiency:

Drug content $=[($ drug weight in the polymeric micelle $) /$ (weight of polymeric micelle) $] \times 100$

Loading efficiency $=[($ Residual drug in the polymeric micelle $) /$ (initial feeding amount of drug) $] \times 100$.

\section{Analysis of polymeric micelles}

Characterization of the polymeric micelles was performed in dimethyl sulfoxide or $\mathrm{D}_{2} \mathrm{O}$ using $500 \mathrm{mHz}{ }^{1} \mathrm{H}-\mathrm{NMR}$ spectroscopy ( $500 \mathrm{mHz}$ superconducting FT-NMR spectrometer, Varian Unity Inova 500 MHz NB High Resolution FT NMR; Varian Inc, Santa Clara, CA). Morphology of the polymeric micelles was observed using a transmission electron microscope (TEM, JEOL JEM-2000 FX II, Japan). One drop of polymeric micelle solution containing phosphotungstic acid $0.05 \%(\mathrm{w} / \mathrm{w})$ was placed onto a carbon film coated on a copper grid for TEM. Observation was done at $80 \mathrm{kV}$. The particle size of the polymeric micelles was measured with dynamic laser scattering (DLS-7000, Otsuka Electronics Company, Osaka, Japan). A sample solution prepared via the dialysis method was used to determine particle size (concentration $1 \mathrm{mg} / \mathrm{mL}$ ).

\section{Drug-release study in vitro}

The release experiment was carried out in vitro as follows. Polymeric micelles incorporating $3 \mathrm{mg}$ of doxorubicin were reconstituted in $5 \mathrm{~mL}$ of phosphate-buffered saline $(0.1 \mathrm{M}$, $\mathrm{pH}$ 7.4), and the ensuing solution was introduced into the dialysis membrane. Next, the dialysis membrane was placed in a $200 \mathrm{~mL}$ bottle with $95 \mathrm{~mL}$ of phosphate-buffered saline. This bottle was placed in a shaking incubator with a stirring speed of $100 \mathrm{rpm}$ at $37^{\circ} \mathrm{C}$. At specific time intervals, the media were sampled for analysis of drug concentration. Afterwards, the whole media was replaced with fresh phosphate-buffered saline to prevent drug saturation. The concentration of doxorubicin released into the phosphate-buffered saline was measured using the ultraviolet spectrophotometer at $479 \mathrm{~nm}$.

\section{Drug cytotoxicity test in vitro}

HuCC-T1 cells (a doxorubicin-sensitive human cholangiocarcinoma cell line) maintained in Dulbecco's modified Eagle's medium $\left(10 \%\right.$ fetal bovine serum, $5 \% \mathrm{CO}_{2}$ at $\left.37^{\circ} \mathrm{C}\right)$ were used to evaluate the antitumor activity of the polymeric micelles. To prepare doxorubicin-resistant HuCC-T1 cells, the HuCC-T1 cells were exposed to doxorubicin for 3 hours and replaced with fresh medium. After that, the cells were incubated for 2 days in the absence of doxorubicin. This process was repeated three times with the same doxorubicin concentrations. The doxorubicin concentration was then gradually increased from $0.0001 \mu \mathrm{g} / \mathrm{mL}$ to $0.1 \mu \mathrm{g} / \mathrm{mL}$ over 2 months.

Viability of the tumor cells was evaluated by an MTT cell proliferation assay. Doxorubicin-resistant HuCC-T1 cells were seeded at a density of $1 \times 10^{4}$ cells 
per well in 96-well plates with $100 \mu \mathrm{L}$ of medium before addition of the polymeric micelles. Next, free doxorubicin, polymeric micelles incorporating doxorubicin, and empty polymeric micelles were added to 96 -well plates at $100 \mu \mathrm{L}$. Controls were treated with $0.5 \% \mathrm{v} / \mathrm{v}$ of dimethyl sulfoxide. After 2 days of incubation, $30 \mu \mathrm{L}$ of MTT $(5 \mathrm{mg} / \mathrm{mL})$ was added to the 96-well plates and incubated for 4 hours. The formazan crystals formed were solubilized with dimethyl sulfoxide, and the absorbance (560 nm test/630 nm reference) was determined using an automated computer-linked microplate reader (Molecular Device Company, Sunnyvale, CA). Each measurement of drug concentration was obtained as the mean value of eight wells. The amount of formazan present is proportional to the number of viable cells, because only living cells will reduce MTT to blue formazan. The results were expressed as a percentage of the absorbance present in the drug-treated cells compared with that in the control cells.

\section{Observation of cells treated with polymeric micelles containing doxorubicin}

Doxorubicin-resistant HuCC-T1 cells were seeded onto cover-glass and treated with doxorubicin or polymeric micelles incorporating doxorubicin. After that, cells were washed with phosphate-buffered saline $(\mathrm{pH} 7.4,0.1 \mathrm{M})$ and were treated with $4 \%$ paraformaldehyde. Next, cells were washed with phosphate-buffered saline and fixed by immobilization solution (ImmuMount, Thermo Electron Corporation, Pittsburgh, PA). These cells were observed with a confocal laser scanning microscope (CLSM, TCS-SP2; Leica, Wetzlar, Germany).

\section{Flow cytometry analysis}

To evaluate the extent of doxorubicin in the cell, doxorubicinresistant HuCC-T1 cells were analyzed using a flow cytometer. Doxorubicin-resistant HuCC-T1 cells were seeded at a density of $1 \times 10^{6}$ cells in 6-well plates and incubated overnight. Free doxorubicin or polymeric micelles incorporating doxorubicin $1 \mu \mathrm{g} / \mathrm{mL}$ were added and incubated for 1 hour. Cells were harvested and analyzed with a flow cytometer. An excitation wavelength at $488 \mathrm{~nm}$ and emission wavelength at $522 \mathrm{~nm}$ were used to observe the fluorescence intensity of doxorubicin.

\section{Statistical analysis}

Wilcoxon statistics and Student's $t$-test (StatGraphics, SigmaPlot) were used to assess the differences between experimental groups. $P<0.05$ was taken as being statistically significant.

\section{Results Characterization of DexbLG block copolymer}

DexbLG copolymer was synthesized using aminated dextran and PLGA-NHS, as shown in Figure 1. Because polysaccharides normally have one reductive end, they are suitable material for block or graft copolymers. The reductive end of dextran was treated with sodium cyanoborohydride. Next, aminated dextran was prepared by coupling with an excess amount of hexamethylene diamine, as shown in Figure 1, and aminated dextran was purified by dialysis. The aminated dextran (dextran- $\mathrm{NH}_{2}$ ) characterized by proton NMR had intrinsic peaks at both dextran (3-5.5 ppm) and hexamethylene diamine (1-2.6 ppm, data not shown). Because the protons at the 1 position of dextran before and after their conjugation with hexamethylene diamine can be assumed to be similar, the ratio of the peak intensity of the protons at the 1 position of dextran to that of hexamethylene diamine was used to estimate the molecular weight and conjugation yield. The conjugation yield of hexamethylene diamine was greater than $98 \%$. Table 1 presents the characterization of dextran and aminated dextran. As shown in Table 1, the molecular weight of the dextran and aminated dextran were measured by gel permeation chromatography. The aminated dextran has a larger molecular weight than that of dextran, although the differences in molecular weight did not exactly reflect the extent of hexamethylene diamine. To conjugate PLGA copolymer, the carboxylic end of PLGA copolymer was activated at the N-hydroxysuccinimide end (PLGA-NHS). PLGA-NHS was mixed with aminated dextran in dry dimethyl sulfoxide to synthesize block copolymer. The synthesized DexbLG block copolymer was purified by dialysis against water and precipitation in methylene chloride. Because the average molecular weight of dextran was less than $5000 \mathrm{~g} / \mathrm{mol}$, free dextran was removed by dialysis against water. Because DexbLG copolymer is not soluble in methylene chloride, unconjugated free PLGA copolymer was removed by precipitation of the synthesized block copolymer into methylene chloride. Figure 2 shows the NMR proton spectra of the resulting block copolymer. As shown in Figure 2, intrinsic peaks of PLGA were observed at $1.5 \mathrm{ppm}$ and $4.8 \mathrm{ppm}$, while dextran showed specific peaks at 3-4 ppm. Table 1 shows the calculated molecular weight of DexbLG, indicating that molecular weight calculated from experimental values was not significantly different from the theoretical values. These results indicated that the DexbLG block copolymer was successfully synthesized. 


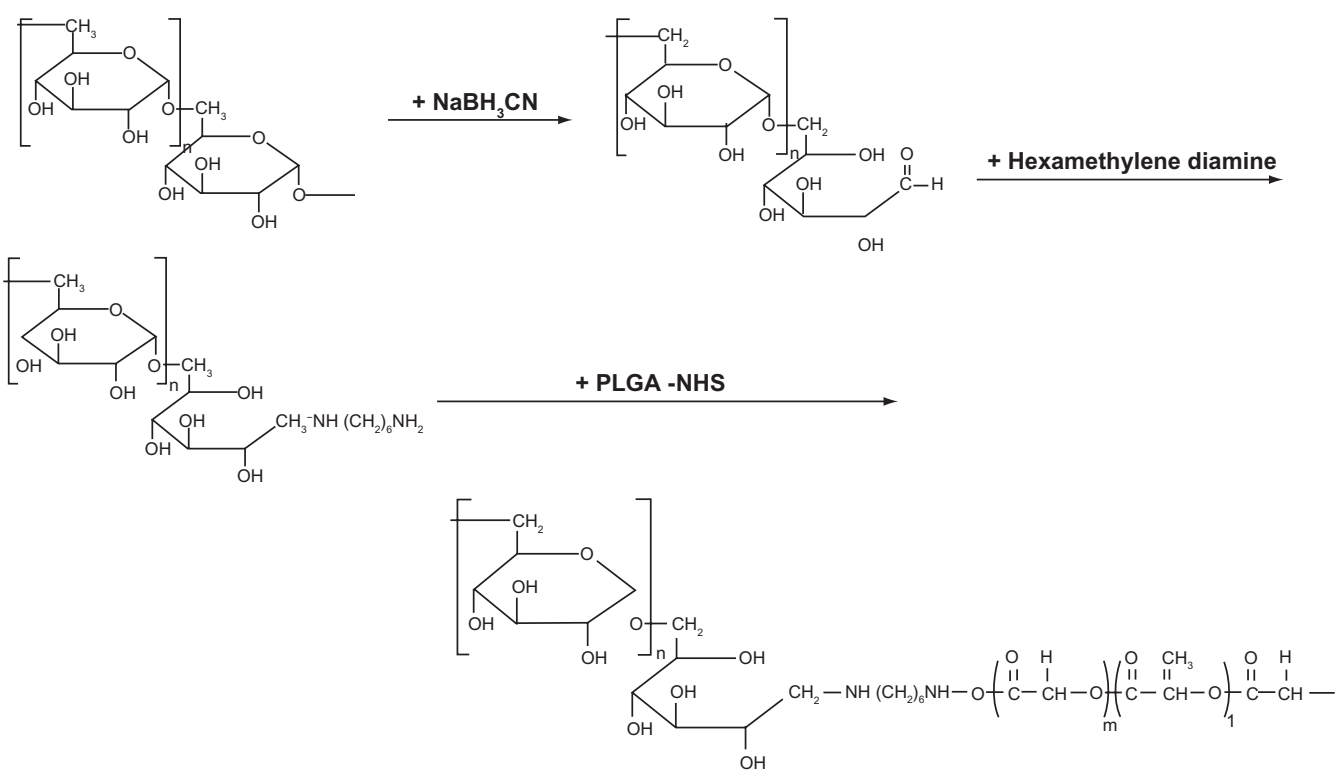

Figure I Synthesis schema for dextran and poly(DL-lactide-co-glycolide) block copolymer.

\section{Characterization of polymeric micelles of DexbLG block copolymer}

Polymeric micelles of DexbLG block copolymer were prepared by dialysis. Because PLGA is hydrophobic, the PLGA block should form the inner core of the polymeric micelle while the dextran domain should form the outer shell. Particle size was measured to investigate whether or not DexbLG block copolymer could form polymeric micelles. As shown in Figure 3, DexbLG block copolymer formed polymeric micelles with a narrow size distribution. The TEM image also demonstrates formation of polymeric micelles smaller than $100 \mathrm{~nm}$. Empty polymeric micelles have an average particle size of approximately $70-80 \mathrm{~nm}$, as shown in Table 2. To make polymeric micelles incorporating the drug, doxorubicin and DexbLG block copolymer were dissolved in dimethyl sulfoxide, and the polymer solution was dropped into deionized water to form polymeric

Table I Characterization of DexbLG block copolymer

\begin{tabular}{|c|c|c|c|c|c|}
\hline & \multicolumn{3}{|c|}{ MW by GPC } & \multicolumn{2}{|c|}{ MW by 'H-NMR } \\
\hline & $M_{w}$ & $M_{n}$ & PDI $^{\mathbf{a}}$ & Theoretical $^{\mathrm{b}}$ & Experimental $^{\mathrm{b}}$ \\
\hline Dextran & 4800 & 4370 & 1.098 & & \\
\hline Dextran- $\mathrm{NH}_{2}$ & 5180 & 4880 & 1.061 & & \\
\hline PLGA & 4920 & 4780 & 1.029 & & \\
\hline DexbLG & & & & 10,100 & 9580 \\
\hline
\end{tabular}

Notes: ${ }^{a P D I}=M_{w} / M_{n}$; 'theoretical value was simply calculated from GPC data as follows: $M_{w}$ of dextran- $\mathrm{NH}_{2}+\mathrm{M}_{\mathrm{w}}$ of PLGA. Experimental value was calculated from 'H NMR data of PLGA and dextran.

Abbreviations: GPC, gel permeation chromatography; PDI, polydispersity index; PLGA, poly(DL-lactide-co-glycolide); DexbLG, dextran and poly(DL-lactideco-glycolide); MW, molecular weight; NMR, nuclear magnetic resonance imaging; dextran- $\mathrm{NH}_{2}$, aminated dextran. micelles via self-aggregation. Solvent and free drug were removed by dialysis against deionized water. These results are summarized in Table 2. As shown in Table 2, drug content was $7 \%-12 \%(\mathrm{w} / \mathrm{w})$ according to the feeding ratio of the drug, and the higher the initial feeding of drug, the higher the drug content. Average particle size increased with an increase in drug content, as shown in Table 2. At a higher drug content, the average particle size of DexbLG polymeric micelles increased above $160 \mathrm{~nm}$.

Amphiphilic macromolecules, such as block and graft copolymer, are able to form polymeric micelles through a selfassembling process in aqueous solution. ${ }^{5,9,13}$ Because dextran is a hydrophilic domain and PLGA is a hydrophobic domain, DexbLG block copolymer also has amphiphilic properties and can form core-shell-type polymeric micelles. A ${ }^{1} \mathrm{H}-\mathrm{NMR}$ spectra study of core-shell-type nanoparticles of DexbLG block copolymer is another way to demonstrate the coreshell structure of nanoparticles. Figure 4 shows the ${ }^{1} \mathrm{H}-\mathrm{NMR}$ spectra of polymeric micelles of DexbLG block copolymer in $\mathrm{D}_{2} \mathrm{O}$ and dimethyl sulfoxide. When polymeric micelles of DexbLG block copolymer were in $\mathrm{D}_{2} \mathrm{O}$, the specific peak of PLGA at $1.5 \mathrm{ppm}$ disappeared, while specific peaks of dextran were clearly found at 3-4.7 ppm (Figure 4A). However, the ${ }^{1} \mathrm{H}-\mathrm{NMR}$ spectra of polymeric micelles of DexbLG block copolymer revealed both peaks of dextran and PLGA in dimethyl sulfoxide, as shown in Figure 4B. These results indicated that DexbLG polymeric micelles have the typical structure of polymeric micelles, ie, PLGA composed of a hydrophobic inner core and dextran composed of a hydrophilic outer shell. Furthermore, the self-assembling property 

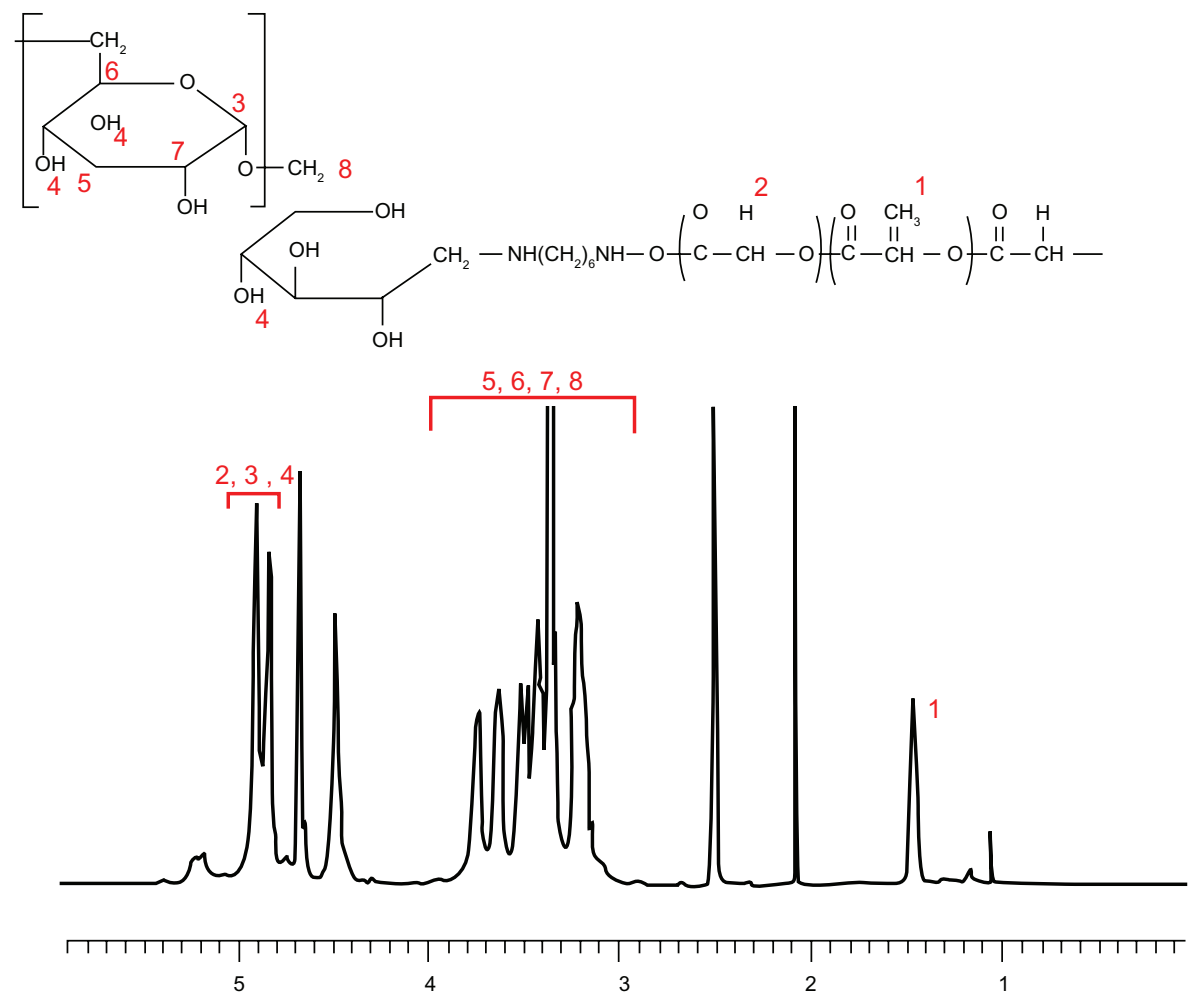

Figure 2 'H-NMR spectra of dextran and poly(DL-lactide-co-glycolide) block copolymer in dimethyl sulfoxide.

of block copolymer is known to depend on the concentration of polymers in aqueous solution. To determine the critical micelle concentration of block copolymer, a fluorescence study was performed using pyrene as the hydrophobic probe. Figure 5A shows the fluorescence excitation spectra of pyrene



Figure 3 Transmission electron micrograph of polymeric micelles composed of dextran and poly(DL-lactide-co-glycolide) block copolymer. at various concentrations of DexbLG block copolymer. The fluorescence intensity was increased according to the increase in polymer concentration, which indicates self-assembly of DexbLG block copolymer in water. In addition, a red shift was observed in the excitation spectra with increasing concentration of DexbLG block copolymer, indicating that pyrene is preferentially partitioned to the hydrophobic inner core of the polymeric micelle. This red shift was presented as the intensity ratio of $I_{339} / I_{336}$ versus log c of DexbLG block copolymer for the pyrene excitation spectra, as shown in Figure 5B. A flat region at extremely low concentration and a sigmoid change in the crossover region were observed. This result indicates that the signal change in the crossover region could be related to the critical micelle concentration value of DexbLG block copolymer. The critical micelle concentration values were found to be $0.00485 \mathrm{~g} / \mathrm{L}$. These results demonstrated that the hydrophilic domain (dextran) of DexbLG block copolymer serves as a flexible outer shell of core-shell structure in aqueous solution, while the hydrophobic domain (PLGA) forms the solid inner core.

Figure 6 shows doxorubicin release from the polymeric micelles. As shown in Figure 6, the higher the drug content, the slower the drug release. An initial burst release of drug was observed for 10 hours, and then doxorubicin 
Table 2 Characterization of DexLG polymeric micelles incorporating doxorubicin

\begin{tabular}{|c|c|c|c|c|c|}
\hline \multirow{2}{*}{$\begin{array}{l}\text { Polymer/doxorubicin } \\
\text { weight ratio }(\mathrm{mg} / \mathrm{mg})\end{array}$} & \multicolumn{2}{|c|}{ Drug contents $(\%, w / w)$} & \multicolumn{3}{|c|}{ Particle size (nm) } \\
\hline & Theoretical & Experimental & $\begin{array}{l}\text { Intensity } \\
\text { (mean } \pm \text { SD) }\end{array}$ & $\begin{array}{l}\text { Weight } \\
(\text { mean } \pm \text { SD })\end{array}$ & $\begin{array}{l}\text { Number } \\
(\text { mean } \pm \text { SD) }\end{array}$ \\
\hline $40 / 0$ & - & - & $82.6 \pm 17.3$ & $75.6 \pm 10.8$ & $70.9 \pm 8.7$ \\
\hline $40 / 5$ & II.I & 7.6 & $\mid 22.1 \pm 29.6$ & $103.3 \pm 24.0$ & $91.4 \pm 17.1$ \\
\hline $40 / 10$ & 20 & 12.3 & $206.5 \pm 49.7$ & $177.5 \pm 38.5$ & $160.2 \pm 27.2$ \\
\hline
\end{tabular}

was continuously released over 4 days. These results indicated that hydrophobic interactions increase at higher drug content, and this phenomenon caused decreased drug release because doxorubicin is a hydrophobic anticancer agent.

\section{In vitro cell cytotoxicity}

To investigate the antitumor activity of free doxorubicin and DexbLG polymeric micelles incorporating doxorubicin, polymeric micelles incorporating doxorubicin were tested with doxorubicin-sensitive HuCC-T1 cells and doxorubicinresistant HuCC-T1 cells. For this test, doxorubicin-resistant HuCC-T1 cells were exposed to doxorubicin $0.0001 \mu \mathrm{g} / \mathrm{mL}$ to $0.1 \mu \mathrm{g} / \mathrm{mL}$ for several months. As shown in Figure 7A, free doxorubicin and polymeric micelles incorporating doxorubicin showed dose-dependent growth inhibition of doxorubicinsensitive HuCC-T1 cells. Cell viability on exposure to free doxorubicin and to polymeric micelles incorporating doxorubicin was not significantly different. However, polymeric micelles incorporating doxorubicin showed higher antitumor cytotoxicity against doxorubicin-resistant HuCC-T1 cells, as shown in Figure 7B. As shown in Figure 7C, the empty polymeric micelles did not significantly inhibit tumor cell growth. The $\mathrm{IC}_{50}$ of polymeric micelles against doxorubicinresistant HuCC-T1 cells was significantly lower than that for free doxorubicin, as shown in Figure 8B, but for doxorubicinsensitive cells was not significantly different. To verify these results, HuCC-T1 cells were observed using confocal laser



Figure 4 ' H-NMR spectra of dextran and poly(DL-lactide-co-glycolide) polymeric micelle in $\mathrm{D}_{2} \mathrm{O}(\mathbf{A})$ and dimethyl sulfoxide (B). 
A

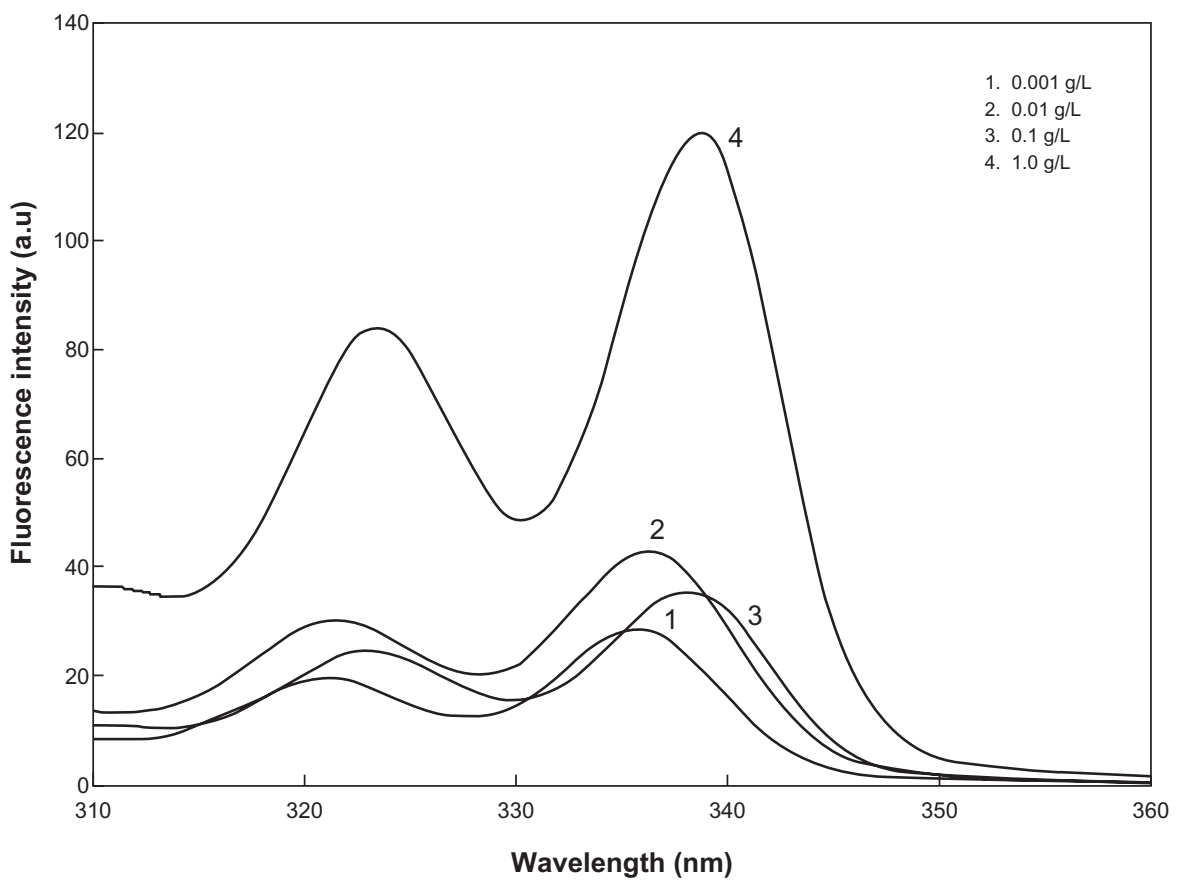

B

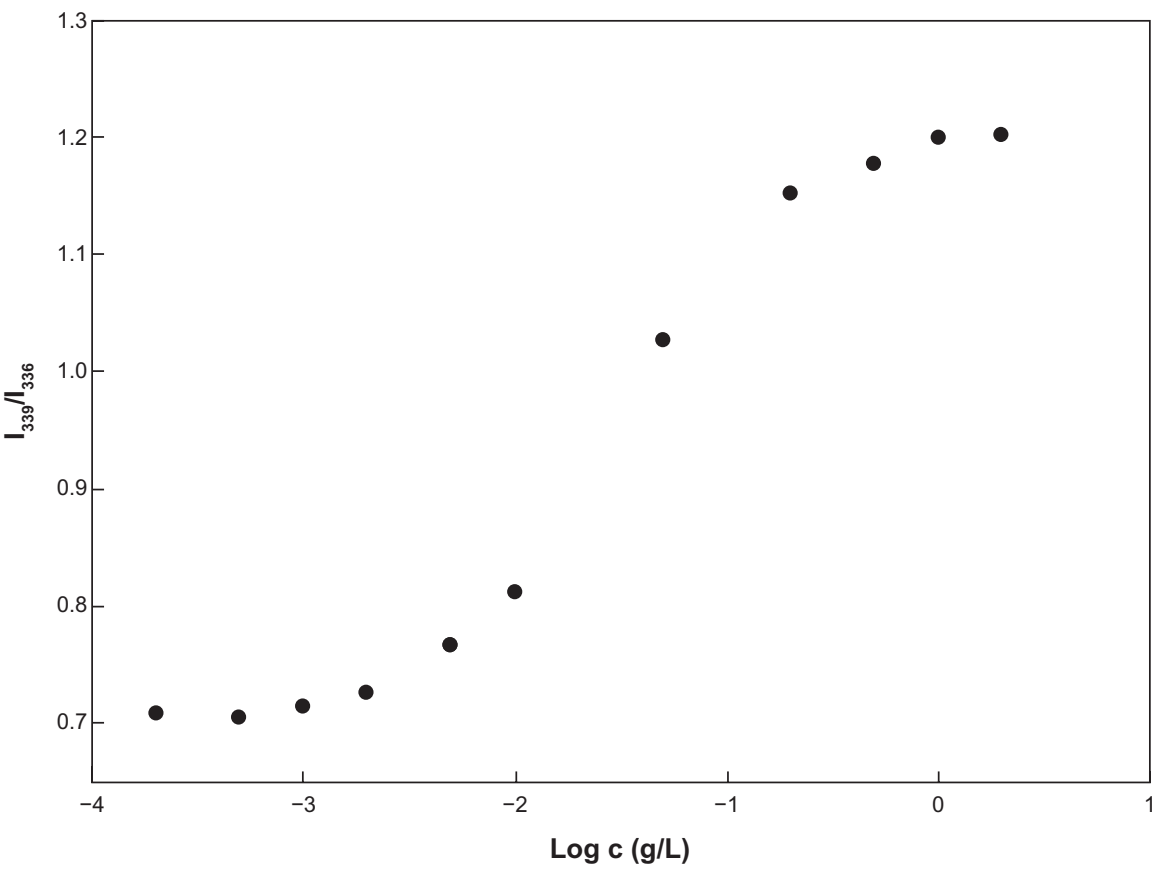

Figure 5 Fluorescence excitation of pyrene $\left(6.0 \times 10^{-7} \mathrm{M}\right)$ versus the concentration of dextran and poly (DL-lactide-co-glycolide) copolymer in distilled water $\left(\lambda_{\mathrm{em}}=390 \mathrm{~nm}\right)$ (A) plots of intensity ratios $I_{339} / I_{336}$ from the pyrene excitation spectra versus (B) $\log \mathrm{c}$ of dextran and poly(DL-lactide-co-glycolide) copolymers in distilled water.

scanning microscopy because doxorubicin has strong red fluorescence, ie, tumor cells will reveal red fluorescence when doxorubicin enters the cell. Figure 9 supports the growth inhibition data. When doxorubicin-resistant cells were treated with free doxorubicin, HuCC-T1 cells revealed very weak red fluorescence intensity, while the polymeric micelles showed strong red fluorescence intensity. Figure 10 also supported these results, ie, polymeric micelle treatment showed higher fluorescence intensity than free doxorubicin treatment. These results showed that DexbLG polymeric micelles incorporating doxorubicin are superior candidates for anticancer drug targeting. 


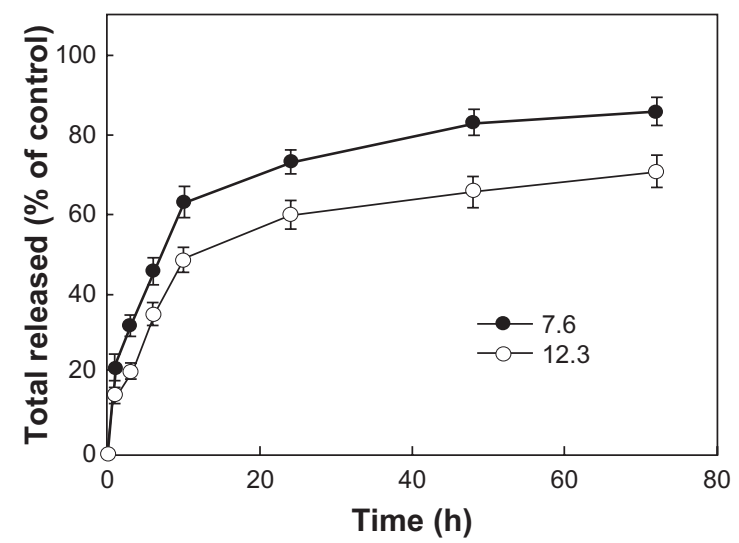

Figure 6 Drug release from polymeric micelles incorporating doxorubicin according to drug content.

\section{Discussion}

Amphiphilic macromolecules, such as block or graft copolymer and hydrophobized polysaccharides, can normally form selfaggregated nanoparticles or polymeric micelles. ${ }^{9-11}$ In particular, block copolymer composed of hydrophilic and hydrophobic domains can form polymeric micelles via self-assembling, ie, the hydrophobic block forms an inner core of the polymeric micelle while the hydrophilic domain forms an outer shell. ${ }^{10,13}$ Polymeric micelles can incorporate anticancer drugs by hydrophobic interaction between the drug and the hydrophobic domain of the block copolymer. ${ }^{12}$ Given these advantages, polymeric micelles have been extensively investigated as delivery vehicles for anticancer drug targeting. Kwon et $\mathrm{al}^{14}$ reported that polymer micelles incorporating anticancer drugs showed enhanced accumulation in tumor cells and prolonged blood circulation times. Because polymeric micelles themselves do not have an active targeting moiety, the mechanism of tumor targeting is referred to as passive targeting by size exclusion or by an enhanced permeation and retention effect. ${ }^{17-19}$ Jeong et a ${ }^{10,20}$ also reported enhanced antitumor activity for polymeric micelles incorporating doxorubicin, reporting longer survival of mice and suppression of tumor growth.

We synthesized block copolymer composed of dextran (hydrophilic block) and PLGA (hydrophobic block). Dextran is a well known biocompatible material, and has been widely used as a bioinert material and drug-carrying material. Feng et $\mathrm{al}^{3}$ reported that dextran plays a positive role in improving lymphatic drainage of liposomes. In addition, carboxymethyl dextrans are known to be superior vehicles for tumor targeting. ${ }^{17}$ Even though dextran is a superior biomaterial, it should be hydrophobically modified to make nanoparticulate vehicles. Poly(ethylene glycol) (PEG), which is frequently used as the hydrophilic domain of polymeric micelles, ${ }^{9,10,12}$ is also a good
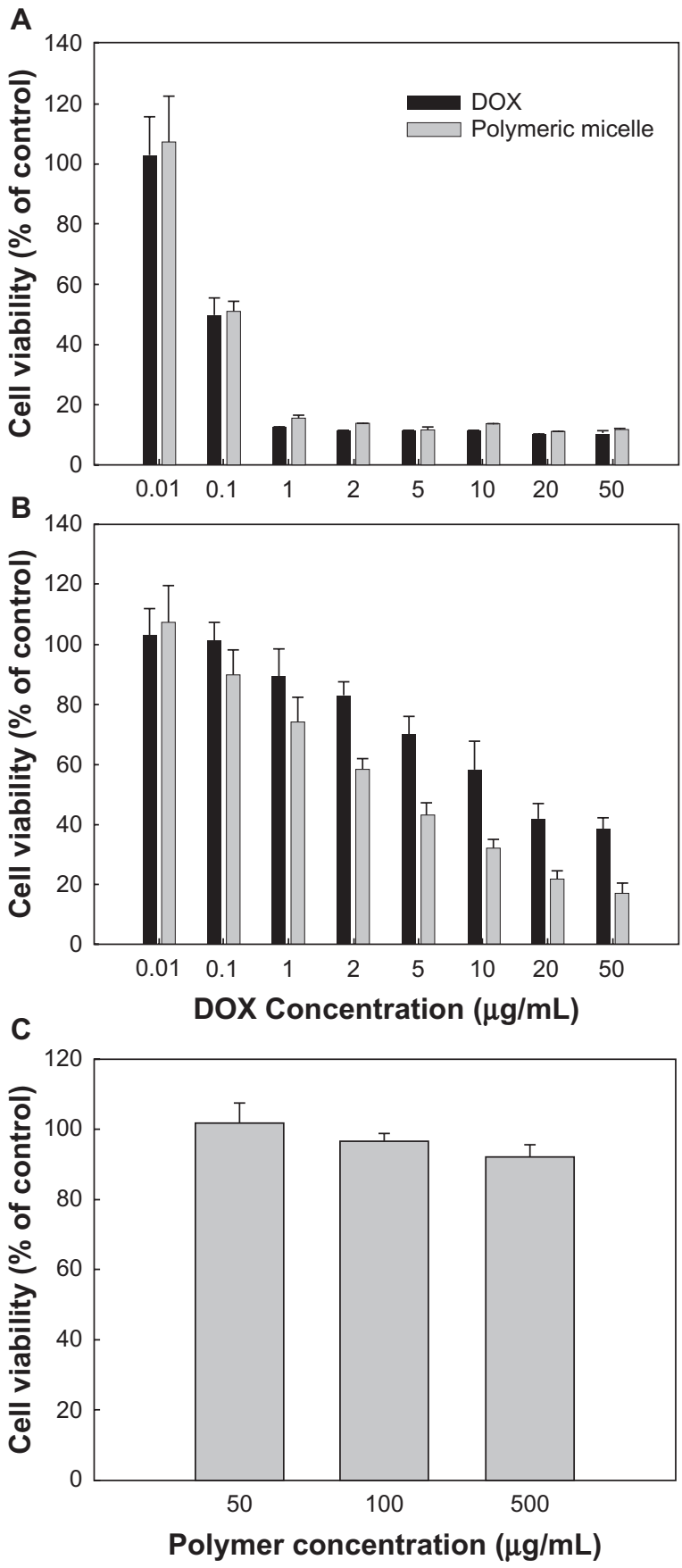

Figure 7 Growth inhibition of doxorubicin-sensitive (A) and doxorubicin-resistant (B) HuCC-TI cells by treatment of polymeric micelles incorporating doxorubicin. $\mathrm{I} \times 10^{4}$ cells were exposed to doxorubicin or polymeric micelles for 2 days. Empty polymeric micelles were treated in a similar way to $(\mathbf{C})$. Empty polymeric micelles did not significantly inhibit cell growth rate.

biomaterial. However, PEG has a limited functional group in its chain, ie, it has only one or two functional groups at the end of the chain. ${ }^{18}$ From this point of view, dextran has an advantage, in that it has a confluent functional (hydroxyl) group in its chain, and the hydroxyl group can be used for chemical modification with targeting moieties, monoclonal antibodies, and drug 

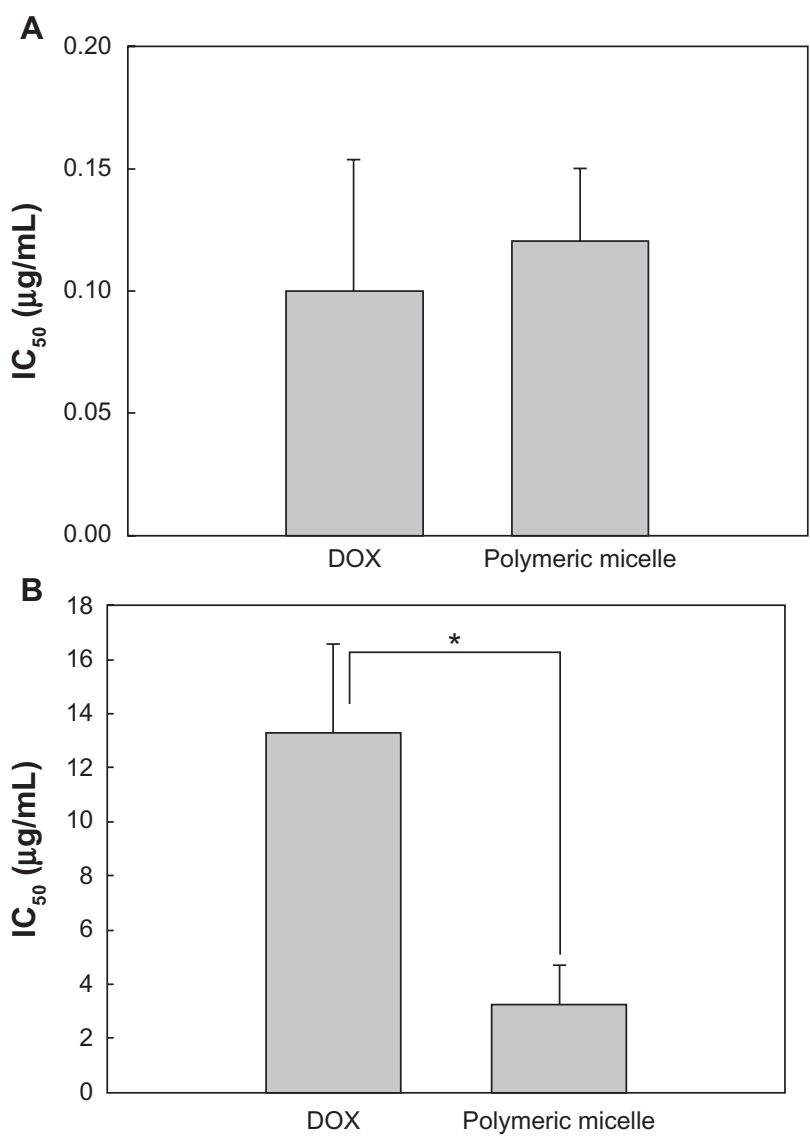

Figure $8 \mathrm{IC}_{50}$ of doxorubicin and polymeric micelles against doxorubicin-resistant HuCC-TI cells.

Notes: $* P<0.01$. The values were averaged for four independent experiments. conjugation. Therefore, the DexbLG block copolymer could be considered an ideal candidate for drug targeting.

Sun et $\mathrm{a}^{22}$ also reported the synthesis of diblock copolymers composed of dextran and poly( $\varepsilon$-caprolactone) (PCL) via sulfide linkage. They argued that reduction-responsive biodegradable micelles can be prepared using dextran-PCL copolymer. Susa et $\mathrm{al}^{23}$ also reported that polymeric nanoparticles prepared from dextran are superior vehicles for overcoming drug resistance in osteosarcoma. DexbLG polymeric micelles have spherical shapes (determined by TEM) and are smaller than $100 \mathrm{~nm}$, as shown in Figure 3. Particle size distribution data also showed a narrow size distribution of DexbLG polymeric micelles. Furthermore, the particle sizes of DexbLG polymeric micelles are favorable for passive drug targeting because nanoparticles smaller than $200 \mathrm{~nm}$ are able to prevent attack by the reticuloendothelial system. ${ }^{24}$ To verify the core shell structure, DexbLG polymeric micelles were measured by ${ }^{1} \mathrm{H}-\mathrm{NMR}$ as shown in Figure 4. In ${ }^{1} \mathrm{H}-\mathrm{NMR}$ spectra, specific peaks for both dextran and PLGA appeared in dimethyl sulfoxide. However, specific peaks of PLGA disappeared in $\mathrm{D}_{2} \mathrm{O}$, while intrinsic peaks of dextran still appeared. These results indicated that DexbLG block copolymer is able to self-aggregate as a spherical, polymeric micelle, and these compounds have a typical micellar structure.

HuCC-T1 cells were used to assess the potential of DexbLG polymeric micelles as anticancer drug delivery vehicles.

\section{DOX-sensitive cell}
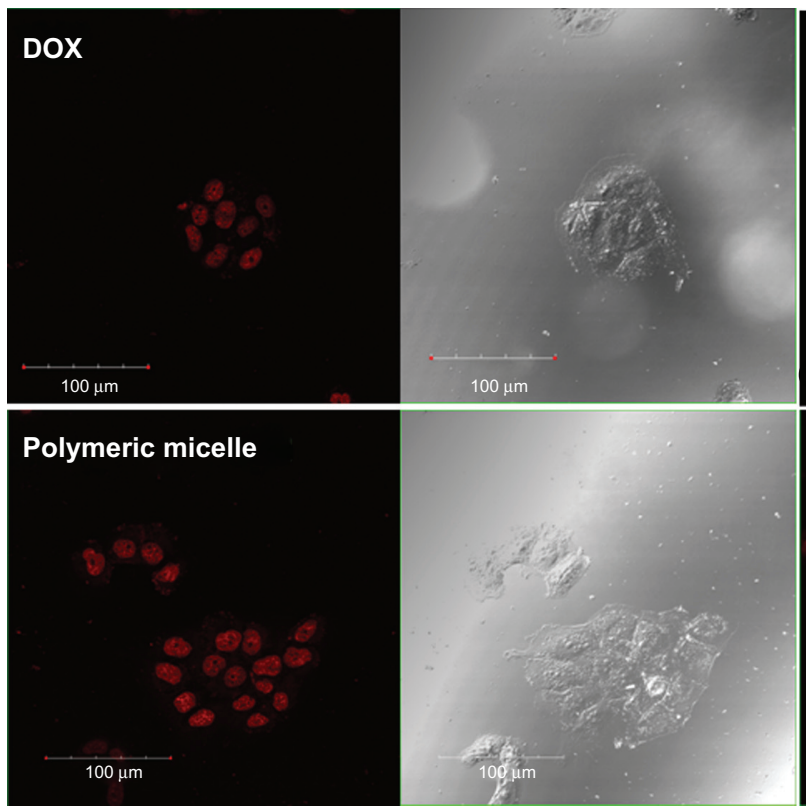

\section{DOX-resistant cell}

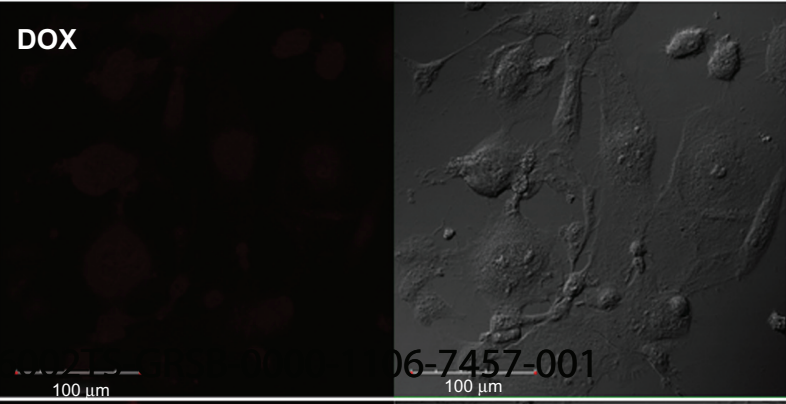

Polymeric micelle



Figure 9 Fluorescence images of doxorubicin-sensitive and doxorubicin-resistant HuCCTI cells. HuCCTI cells were exposed to doxorubicin or polymeric micelles (equivalent concentration of doxorubicin I $\mu \mathrm{g} / \mathrm{mL}$ ) for I hour. Fluorescence images of cells were observed with confocal laser scanning microscopy. 



Figure 10 Flow cytometric analysis of doxorubicin-sensitive and doxorubicin-resistant HuCC-TI cells. HuCCTI cells were exposed to doxorubicin or polymeric micelles (equivalent concentration of doxorubicin I $\mu \mathrm{g} / \mathrm{mL}$ ) for I hour. I × $10^{6}$ cells were used for FACScan analysis. Doxorubicin-sensitive cells: (A) control, (B) doxorubicin, $(\mathbf{C})$ polymeric micelles, (D) relative fluorescence intensity. Doxorubicin-resistant cells: (E) control; $(\mathbf{F})$ doxorubicin; $(\mathbf{G})$ polymeric micelles; $(\mathbf{H})$ relative fluorescence intensity. The values were averaged over four separate experiments.

Notes: $* P<0.005 ; * * P<0.0001$; $* * * P<0.0001 ; * * * * P<0.0001$.

Prior to testing for anticancer activity, doxorubicin-resistant HuCC-T1 cells were prepared. To generate doxorubicinresistant HuCC-T1 cells, tumor cells were exposed to doxorubicin at a concentration of $0.0001 \mu \mathrm{g} / \mathrm{mL}$, and replaced with fresh medium. The doxorubicin concentration gradually increased to $0.1 \mu \mathrm{g} / \mathrm{mL}$. This procedure is a simple way to create doxorubicin-resistant tumor cells. ${ }^{10}$ Doxorubicin-resistant tumor cells are normally resistant to 
penetration of doxorubicin through the cell membrane and resistant to doxorubicin toxicity. ${ }^{10}$ However, nanoparticles or polymeric micelles are known to overcome this barrier and efficiently deliver the drug to the tumor cell. ${ }^{10,23,25}$ As shown in Figure 7, free doxorubicin and polymeric micelles containing doxorubicin dose-dependently inhibited growth of doxorubicin-sensitive HuCC-T1 cells. However, doxorubicin-resistant cells had higher survival rates after doxorubicin treatment than did doxorubicin-sensitive cells. Even though polymeric micelles targeted to doxorubicinresistant cells also showed lower cytotoxicity than that of doxorubicin-sensitive cells (Figure 7B), they had higher antitumor activity than that of free doxorubicin. These results indicated that doxorubicin-resistant $\mathrm{HuCC}$-T1 cells are resistant to doxorubicin. However, polymeric micelles have higher potency against doxorubicin-resistant HuCC-T1 cells than free doxorubicin, indicating that polymeric micelles can penetrate the cell membrane and are engulfed by the cells while free doxorubicin barely penetrated the cell membrane. To verify this hypothesis, doxorubicin and polymeric micelle-treated HuCC-T1 cells were observed with confocal laser scanning microscopy because doxorubicin itself expresses strong red fluorescence. Confocal laser scanning microscopy data supported these phenomena, as shown in Figure 9. Doxorubicin-sensitive cells, doxorubicin, and polymeric micelles showed also similar fluorescence images. However, fluorescence intensity for doxorubicin-resistant cells was very weak upon treatment with free doxorubicin, while polymeric micelles expressed strong red fluorescence. Flow cytometric measurement of HuCC-T1 cells further supported these phenomena, as shown in Figure 10. The fluorescent intensity of polymeric micelle treatment was almost double that of doxorubicin treatment. These results indicated that polymeric micelles of DexbLG are helpful for overcoming drug resistance of tumor cells. Susa et $\mathrm{al}^{23}$ also reported that dextran nanoparticles encapsulating doxorubicin had a curative effect on multidrug resistant osteosarcoma cell lines by increasing the amount of drug accumulation in the nucleus via a P glycoprotein-independent pathway. They argued that dextran nanoparticles are effective for increasing apoptosis of tumor cells. Furthermore, Gao et $\mathrm{al}^{25}$ reported that nanoparticles incorporating doxorubicin using dextran/1,2-dipalmitoylsn-glycero-3-phosphoethanolamine copolymer exhibited higher antitumor activity than that of doxorubicin itself against HeLa cells. We also observed the enhanced delivery of polymeric micelles to doxorubicin-resistant HuCC-T1 cells. We suggest that polymeric micelles of
DexbLG are promising candidates for antitumor drug targeting.

\section{Conclusion}

DexbLG block copolymer was synthesized to make polymeric micelles incorporating doxorubicin. Polymeric micelles from DexbLG block copolymer are spherical and smaller than $100 \mathrm{~nm}$. The particle size of the polymeric micelles incorporating doxorubicin increased with increasing drug content. Higher initial drug feeding induced higher drug content and a slower drug release rate. Drug release decreased with an increase of drug content. In an in vitro antiproliferation study, polymeric micelles were more effective against doxorubicin-resistant HuCC-T1 cells than free doxorubicin, indicating that polymeric micelles were effectively engulfed by tumor cells, while free doxorubicin barely penetrated the tumor cell membrane. These results were supported by confocal laser scanning microscopic observation, ie, free doxorubicin treatment showed very weak fluorescence intensity while polymeric micelle treatment showed strong red fluorescence. Flow cytometric analysis of tumor cells also supported these results. Polymeric micelle treatment showed twice the fluorescence intensity as that from doxorubicin treatment. We suggest that DexbLG polymeric micelles incorporating doxorubicin are promising candidate vehicles for antitumor drug targeting.

\section{Acknowledgment}

This study was supported by a grant of the Korea Healthcare Technology R\&D Project, Ministry for Health and Welfare, Republic of Korea (A091047).

\section{Disclosure}

The authors report no conflicts of interest in this work.

\section{References}

1. Danhauser-Riedl S, Hausmann E, Schick H, et al. Phase I clinical and pharmacokinetic trial of dextran conjugated doxorubicin (AD-70, doxorubicin-OXD). Invest New Drugs. 1993;11:187-195.

2. Du YZ, Weng Q, Yuan H, et al. Synthesis and antitumor activity of stearate-g-dextran micelles for intracellular doxorubicin delivery. ACS Nano. 2010;4:6894-6902.

3. Feng L, Zhang L, Liu M, et al. Roles of dextrans on improving lymphatic drainage for liposomal drug delivery system. J Drug Target. 2010;18:168-178.

4. Ichinose K, Tomiyama N, Nakashima M, et al. Antitumor activity of dextran derivatives immobilizing platinum complex (II). Anticancer Drugs. 2000;11:33-38.

5. Jeong YI, Choi KC, Song CE. Doxorubicin release from core-shell type nanoparticles of poly(DL-lactide-co-glycolide)-grafted dextran. Arch Pharm Res. 2006;29:712-719.

6. Alahdad Z, Ramezani R, Aminlari M, et al. Preparation and properties of dextran sulfate-lysozyme conjugate. J Agric Food Chem. 2009;57: $6449-6454$. 
7. Bisht S, Maitra A. Dextran-doxorubicin/chitosan nanoparticles for solid tumor therapy. Wiley Interdiscip Rev Nanomed Nanobiotechnol. 2009; 1:415-425.

8. Woitiski CB, Neufeld RJ, Veiga F, et al. Pharmacological effect of orally delivered insulin facilitated by multilayered stable nanoparticles. Eur J Pharm Sci. 2010;41:556-563.

9. Gref R, Minamitake Y, Peracchia MT, et al. Biodegradable longcirculating polymeric nanospheres. Science. 1994;263:1600-1603.

10. Jeong YI, Jin SG, Kim IY, et al. Doxorubicin-incorporated nanoparticles composed of poly(ethylene glycol)-grafted carboxymethyl chitosan and antitumor activity against glioma cells in vitro. Colloids Surf B Biointerfaces. 2010;79:149-155.

11. La SB, Okano T, Kataoka K, et al. Preparation and characterization of the micelle-forming polymeric drug indomethacin-incorporated poly(ethylene oxide)-poly(beta-benzyl L-aspartate) block copolymer micelles. J Pharm Sci. 1996;85:85-90.

12. Kwon GS, Naito M, Yokoyama M, et al. Physical entrapment of adriamycin in AB block copolymer micelles. Pharm Res. 1995;12:192-195.

13. Kwon GS, Naito M, Yokoyama M, et al. Polymeric micelles based on $\mathrm{AB}$ block copolymers of poly(ethylene oxide) and poly( $\beta$-benzyl L aspartate). Langmuir. 1993;9:945-949.

14. Kwon GS, Yokoyama M, Okano T, et al. Biodistribution of micelleforming polymer-drug conjugates. Pharm Res. 1993;10:970-974.

15. Maruyama A, Watanabe H, Ferdous A, et al. Characterization of interpolyelectrolyte complexes between double-stranded DNA and polylysine comb-type copolymers having hydrophilic side chains. Bioconjug Chem. 1998;9:292-299.

16. Perez Y, Valdivia A, Ramirez HL, Villalonga R. Improved pharmacokinetics properties for catalase by site-specific glycosidation with aminated dextran. Macromol Rapid Commun. 2005;26(16);1304-1308.
17. Greish K, Nagamitsu A, Fang J, Maeda H. Copoly(styrene-maleic acid)-pirarubicin micelles: high tumor-targeting efficiency with little toxicity. Bioconjug Chem. 2005;16:230-236.

18. Maeda H, Wu J, Sawa T, Matsumura Y, Hori K. Tumor vascular permeability and the EPR effect in macromolecular therapeutics: a review. J Control Release. 2000;65:271-284.

19. Noguchi Y, Wu J, Duncan R, et al. Early phase tumor accumulation of macromolecules: a great difference in clearance rate between tumor and normal tissues. Jpn J Cancer Res. 1998;89:307-314.

20. Jeong YI, Na HS, Cho KO, et al. Antitumor activity of adriamycinincorporated polymeric micelles of poly(gamma-benzyl L-glutamate)/ poly(ethylene oxide). Int J Pharm. 2009;365:150-156.

21. Sugahara S, Kajiki M, Kuriyama H, et al. Paclitaxel delivery systems: the use of amino acid linkers in the conjugation of paclitaxel with carboxymethyldextran to create prodrugs. Biol Pharm Bull. 2002;25:632-641.

22. Sun H, Guo B, Li X, et al. Shell-sheddable micelles based on dextranSS-poly(epsilon-caprolactone) diblock copolymer for efficient intracellular release of doxorubicin. Biomacromolecules. 2010;11:848-854.

23. Susa M, Iyer AK, Ryu K, et al. Doxorubicin loaded polymeric nanoparticulate delivery system to overcome drug resistance in osteosarcoma. BMC Cancer. 2009;9:399.

24. Allemann E, Gurny R, Doelker E. Drug-loaded nanoparticles preparation methods and drug targeting issues. Eur J Pharm Biopharm. 1993;39:173-191.

25. Gao H, Li M, Wu Y. Novel amphiphilic dextran copolymers nanoparticles for delivery of doxorubicin. J Appl Polym Sci. 2010;120:2448-2458.
International Journal of Nanomedicine

\section{Publish your work in this journal}

The International Journal of Nanomedicine is an international, peerreviewed journal focusing on the application of nanotechnology in diagnostics, therapeutics, and drug delivery systems throughout the biomedical field. This journal is indexed on PubMed Central,



\section{Dovepress}

Journal Citation Reports/Science Edition, EMBase, Scopus and the Elsevier Bibliographic databases. The manuscript management system is completely online and includes a very quick and fair peer-review system, which is all easy to use. Visit http://www.dovepress.com/ testimonials.php to read real quotes from published authors. 